\title{
Investigation of electrical potential and electromagnetic field for overhead high voltage power lines in Malaysia
}

\begin{abstract}
The exposure of human body to electric field and magnetic field could cause biological effects, including changes in functions of cells and tissues and subtle changes in hormone levels, which may or may not be harmful. The aim of this study was to analyze and compute the amount of electrical potential, electric field and magnetic flux density at a certain point and distance from the overhead high voltage power lines of 132 and $275 \mathrm{kV}$ in Malaysia. An analytical calculus method is proposed in order to accomplish this study. The models of the power lines were constructed using the actual physical dimensions of the towers. The results show that the exposure levels of the electromagnetic fields to the public is low if they stay more than $30 \mathrm{~m}$ away from the power lines. For the live-line worker, the exposure to the high electric and magnetic field could endanger their body if they stay too close to the conductor. The evaluations of the electrical potential, electric field strength and magnetic flux density are done using the Matlab environment. Matlabô Graphical User Interface (GUI) techniques are developed as an easy and user-friendly tool to be used.
\end{abstract}

Keyword: Electric field; Magnetic field; Power lines; Exposure; Live-line worker; Matlab 